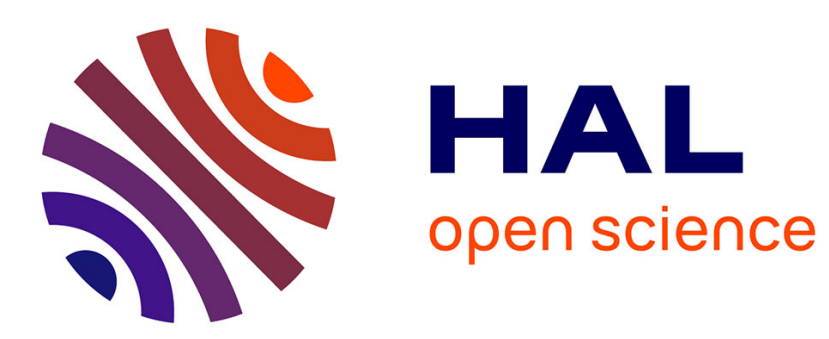

\title{
Quadratic stability for hybrid systems with nested saturations
}

Mirko Fiacchini, Sophie Tarbouriech, Christophe Prieur

\section{To cite this version:}

Mirko Fiacchini, Sophie Tarbouriech, Christophe Prieur. Quadratic stability for hybrid systems with nested saturations. IEEE Transactions on Automatic Control, 2012, 57 (7), pp.1832-1838. 10.1109/TAC.2011.2178651 . hal-00711862

\section{HAL Id: hal-00711862 \\ https://hal.science/hal-00711862}

Submitted on 26 Jun 2012

HAL is a multi-disciplinary open access archive for the deposit and dissemination of scientific research documents, whether they are published or not. The documents may come from teaching and research institutions in France or abroad, or from public or private research centers.
L'archive ouverte pluridisciplinaire HAL, est destinée au dépôt et à la diffusion de documents scientifiques de niveau recherche, publiés ou non, émanant des établissements d'enseignement et de recherche français ou étrangers, des laboratoires publics ou privés. 


\title{
Quadratic stability for hybrid systems with nested saturations
}

\author{
Mirko Fiacchini, Sophie Tarbouriech and Christophe Prieur
}

\begin{abstract}
The problems of characterizing quadratic stability and computing an estimation of the domain of attraction for saturated hybrid systems are addressed. Hybrid systems presenting saturations and nested saturations on signals involved in both the continuous-time and the discrete-time dynamics are considered. Geometrical characterizations of local and global quadratic stability are provided. Computation oriented conditions for quadratic stability are given in form of convex constraints.
\end{abstract}

Index Terms-Hybrid systems, nested saturations, domain of attraction, stability.

\section{INTRODUCTION}

Hybrid systems are systems with both continuous-time and discrete-time dynamics. Recently, the interest on hybrid systems has been growing, see [1], [2], [3], [4], [5], mainly due to the increasing application of digital devices for the control of real systems, like chemical processes, communications and automotive systems. A proper analysis and control theory has to be developed for hybrid systems. See for instance [6], concerning the design of predictive controllers for hybrid systems, and [7], on the use of hybrid controllers to improve the performance.

In this paper, hybrid systems with nested saturations are handled and both local and global stability are considered. The attention is devoted to quadratic Lyapunov functions and ellipsoidal contractive sets, as estimations of the domain of attraction for hybrid systems with (nested) saturations. Considering ellipsoids entails some conservativeness with respect to other families of sets (as polytopes), but permits to pose the problem in an efficiently solvable form. The issue of estimating the domain of attraction for saturated systems, in continuous-time and discrete-time, has been dealt with considering ellipsoids [8], [9], [10], [11], and polytopes [12].

A first contribution of the paper is the geometrical characterization of saturated functions. It is proved that, given a state, its image through a saturated function is contained in a known state-dependent polytope. The property is also proved for the case of nested saturations. Such results permit to characterize contractiveness of ellipsoids and to determine quadratic Lyapunov functions by means of convex constraints. Some

This work was partially supported by the ANR project ArHyCo, ARPEGE, contr. num. ANR-2008 SEGI 004 01-30011459.

M. Fiacchini and S. Tarbouriech are with CNRS; LAAS; 7 avenue du colonel Roche, F-31077 Toulouse, France, Université de Toulouse; UPS, INSA, INP, ISAE, UT1, UTM, LAAS; F-31077 Toulouse, France. ffiacchini, sophie.tarbouriech\}@laas.fr.

C. Prieur is with Department of Automatic Control, Gipsa-lab, Domaine universitaire, 961 rue de la Houille Blanche, BP 46, 38402 Grenoble Cedex, France. christophe.prieurdgipsa-lab.grenoble-inp.fr. results present in literature for continuous-time, as [9], [11], and discrete-time saturated systems, see [10], are improved or recovered as particular cases of our approach, see also the preliminary version of the work [13]. The results on local and global quadratic stability for hybrid systems with simple and nested saturations are other contributions. We also present how the lower bound on the time interval between jumps can be used in the formulation of the stability conditions. Finally, computation oriented conditions for local and global quadratic stability are stated and applied to numerical examples.

Notation. Given $n \in \mathbb{N}$, denote $\mathbb{N}_{n}=\{x \in \mathbb{N}: 1 \leq x \leq n\}$. Given $A \in \mathbb{R}^{n \times m}, A_{i}$ with $i \in \mathbb{N}_{n}$ denotes its $i$-th row, $A_{(j)}$ with $j \in \mathbb{N}_{m}$ its $j$-th column and $A_{i, j}$ the entry of the $i$-th row and $j$-th column of $A$. The identity matrix of order $n$ is denoted $I_{n}$, the null $m \times n$ matrix is $0_{m \times n}$. Given the matrix $P=P^{T}>0$, define the ellipsoid $\mathscr{E}(P)=\left\{x \in \mathbb{R}^{n}: x^{T} P x \leq 1\right\}$. Given the set $D$ and $\alpha \geq 0$, denote the set $\alpha D=\{\alpha x: x \in D\}$ and $\operatorname{co}(D)$ is its convex hull. Given $J \subseteq \mathbb{N}_{m}$, we denote $\bar{J}=\mathbb{N}_{m} \backslash J$, with $m \in \mathbb{N}$. The symbol $*$ stands for symmetric block.

\section{Problem StATEMEnT}

Consider the closed-loop saturated hybrid system, represented by using the hybrid framework introduced in [3], whose continuous-time dynamics is given by

$$
\left\{\begin{aligned}
\dot{x} & =\hat{g}(x)=\hat{A} x+\hat{B} \varphi(\hat{K} x), \\
\dot{\tau} & =1,
\end{aligned}\right.
$$

valid if $(x, \tau) \in \mathscr{F}$, with $x \in \mathbb{R}^{n}$, and discrete-time dynamics

$$
\left\{\begin{array}{l}
x^{+}=\tilde{g}(x)=\tilde{A} x+\tilde{B} \varphi(\tilde{K} x), \\
\tau^{+}=0,
\end{array}\right.
$$

if $(x, \tau) \in \mathscr{J}$. Regions $\mathscr{F}$ and $\mathscr{J}$ are referred to as the flow and jump sets, respectively. Function $\varphi: \mathbb{R}^{a} \rightarrow \mathbb{R}^{a}$ denotes the saturation, i.e. $\varphi_{i}(y)=\operatorname{sgn}\left(y_{i}\right) \min \left\{\left|y_{i}\right|, 1\right\}$, for all $i \in \mathbb{N}_{a}$, with $y \in \mathbb{R}^{a}$. The saturation bounds are assumed equal to 1 , without loss of generality. Sets $\mathscr{F}$ and $\mathscr{J}$ are given by

$$
\begin{aligned}
& \mathscr{F}=\left\{(x, \tau) \in \mathbb{R}^{n+1}: x^{T} M x \geq 0, \quad \text { or } \tau<\rho\right\}, \\
& \mathscr{J}=\left\{(x, \tau) \in \mathbb{R}^{n+1}: x^{T} M x \leq 0, \text { and } \tau \geq \rho\right\},
\end{aligned}
$$

where $M=M^{T} \in \mathbb{R}^{n \times n}$ and $\rho \geq 0$, as in [14]. Different kinds of flow and jump regions can be defined by (3), as the reset conditions used in reset control as studied in [15], [16]. Furthermore, choosing $M=M^{T}>0$ (or $M=M^{T}<0$ ), the formulation (3) permits to restrict the dynamics to a continuous-time (resp. discrete-time) system, see also [13].

Remark 1: The variable $\tau$ represents the time passed from the last jump. Its introduction, together with the parameter 
$\rho \geq 0$, permits to define a lower bound on the interval between two successive jumps. Such a bound, referred to as "temporal regularization", can be used to prevent having an infinite number of jumps in a finite time interval, i.e. Zeno solutions [3], which should be avoided in real applications. Notice that conditions on the state $x$ ensuring the system flowing for a certain amount of time, used in some applications, consist in determining implicitly a positive value of $\rho$. Hereafter the knowledge of $\rho$ is used to allow the potential Lyapunov function to increase during a jump. This leads to more general results than those obtained imposing its decreasing during both the flow and the jumps. This case is recovered by posing $\rho=0$.

Nested saturations are also considered to obtain a more general model (see [17]). In fact, the presence of a further saturation on the plant output is a realistic assumption, as bounds on the measurements are often present. In such case, the continuous dynamics of the hybrid system becomes

$$
\left\{\begin{array}{l}
\dot{x}=\hat{g}(x)=\hat{A} x+\hat{B} \varphi(\hat{K} x+\hat{E} \varphi(\hat{F} x)), \\
\dot{\tau}=1,
\end{array}\right.
$$

and, analogously, the discrete dynamics is

$$
\left\{\begin{array}{l}
x^{+}=\tilde{g}(x)=\tilde{A} x+\tilde{B} \varphi(\tilde{K} x+\tilde{E} \varphi(\tilde{F} x)) \\
\tau^{+}=0 .
\end{array}\right.
$$

The objectives of the paper can be summarized as follows.

Problem 1: Given the flow and jump sets, $\mathscr{F}$ and $\mathscr{J}$, determine an ellipsoidal region $\Omega=\mathscr{E}(P)$, with $P=P^{T}>0$, as large as possible, such that a Lyapunov function in $\Omega$ for the saturated hybrid system (1)-(3), or for the hybrid system with nested saturations (3)-(5), can be determined.

\section{QUADRATIC STABILITY FOR SATURATED HYBRID SYSTEMS}

Hereafter we prove that the image of the state $x$ through a saturated function $g(x)$ is contained within a set explicitly obtainable. This result permits to geometrically characterize quadratic stability for saturated hybrid systems.

Definition 1: Given $D \subseteq \mathbb{R}^{n}$, the support function of $D$ at $\eta \in \mathbb{R}^{n}$ is $\phi_{D}(\eta)=\sup _{x \in D} \eta^{\bar{T}} x$.

Among the properties of support function, see [18], [19], we have that set inclusion conditions can be given in terms of linear inequalities involving the support functions.

Property 1: Given a closed, convex set $D \subseteq \mathbb{R}^{n}$, then $x \in D$ if and only if $\eta^{T} x \leq \phi_{D}(\eta)$, for all $\eta \in \mathbb{R}^{n}$. Given also $C \subseteq \mathbb{R}^{n}$, then $C \subseteq D$ if and only if $\phi_{C}(\eta) \leq \phi_{D}(\eta)$, for all $\eta \in \mathbb{R}^{n}$.

\section{A. Convex bounds of saturated functions}

The following theorem, stated for functions $g(x)=A x+$ $B \varphi(K x)$, with $A \in \mathbb{R}^{n \times n}, B \in \mathbb{R}^{n \times m}$ and $K \in \mathbb{R}^{m \times n}$, can be used to prove results for both the continuous-time and the discrete-time dynamics, and then applied to hybrid systems.

Theorem 1: Given a function $g(x)=A x+B \varphi(K x)$, the ellipsoid $\Omega=\mathscr{E}(P)$, with $P \in \mathbb{R}^{n \times n}$ and $P=P^{T}>0$, and $H(i, J) \in \mathbb{R}^{1 \times n}$ such that $|H(i, J) x| \leq 1$ for all $x \in \Omega$, for every $J \subseteq \mathbb{N}_{m}$ and $i \in J$, then we have $g(x) \in G(x)$ for all $x \in \Omega$, with

$$
G(x)=\operatorname{co}\left(\left\{N(J) x \in \mathbb{R}^{n}: J \subseteq \mathbb{N}_{m}\right\}\right),
$$

and

$$
N(J)=A+\sum_{i \in \bar{J}} B_{(i)} K_{i}+\sum_{i \in J} B_{(i)} H(i, J) .
$$

Proof: Consider $x \in \Omega$. First notice that, given $J \subseteq \mathbb{N}_{m}$ and $i \in J$, we have that $|H(i, J) x| \leq 1$ implies that $\varphi_{i}(K x) \in$ $\operatorname{co}\left(\left\{K_{i} x, H(i, J) x\right\}\right)$. In fact, supposing that $K_{i} x \geq 0$, (case $K_{i} x<0$ is analogous), if $K_{i} x \leq 1$ then $\varphi_{i}(K x)=K_{i} x$. If $K_{i} x>1$ then $\varphi_{i}(K x)=1$ and $H(i, J) x \leq \varphi_{i}(K x)<K_{i} x$, therefore the inclusion is satisfied. This implies that

$$
\eta^{T} B_{(i)} \varphi_{i}(K x) \in \operatorname{co}\left(\left\{\eta^{T} B_{(i)} K_{i} x, \eta^{T} B_{(i)} H(i, J) x\right\}\right) \subseteq \mathbb{R},
$$

holds for all $\eta \in \mathbb{R}^{n}$, every $J \subseteq \mathbb{N}_{m}$ and every $i \in J$. This means that, for every $\eta \in \mathbb{R}^{n}$, we have

$$
\begin{gathered}
\min \left\{\eta^{T} B_{(i)} K_{i} x, \eta^{T} B_{(i)} H(i, J) x\right\} \leq \eta^{T} B_{(i)} \varphi_{i}(K x) \\
\leq \max \left\{\eta^{T} B_{(i)} K_{i} x, \eta^{T} B_{(i)} H(i, J) x\right\} .
\end{gathered}
$$

Hence for any $\eta \in \mathbb{R}^{n}$, every $J \subseteq \mathbb{N}_{m}$ and every $i \in J$, an accurate choice between the values $\eta^{T} B_{(i)} H(i, J) x$ and $\eta^{T} B_{(i)} K_{i} x$ provides an upper bound of $\eta^{T} B_{(i)} \varphi_{i}(K x)$. Thus, given $\eta \in \mathbb{R}^{n}$ and $x \in \Omega$, there exists $J(x, \eta) \subseteq \mathbb{N}_{m}$ such that

$$
\begin{aligned}
\eta^{T} g(x) & =\eta^{T} A x+\sum_{i \in \mathbb{N}_{m}} \eta^{T} B_{(i)} \varphi_{i}(K x) \\
& \leq \eta^{T} A x+\sum_{i \in \bar{J}(x, \eta)} \eta^{T} B_{(i)} K_{i} x+\sum_{i \in J(x, \eta)} \eta^{T} B_{(i)} H(i, J(x, \eta)) x,
\end{aligned}
$$

holds. Hence the support function of $N(J(x, \eta)) x \in G(x)$ is greater than or equal to the support function of $g(x)$, then $\eta^{T} g(x) \leq \phi_{G(x)}(\eta)$. From Property 1, we have $g(x) \in G(x)$.

The meaning of Theorem 1 is that, for all $x \in \Omega$, the image $g(x)$ is contained in the polytope $G(x)$, whose vertices are known. Its extension to functions presenting nested saturations, that is $g(x)=A x+B \varphi(K x+E \varphi(F x))$, with $E \in \mathbb{R}^{m \times p}$ and $F \in \mathbb{R}^{p \times n}$, applies then to both discrete-time and continuoustime systems with nested saturations, as well as to hybrid ones.

Theorem 2: Given a function $g(x)=A x+B \varphi(K x+$ $E \varphi(F x)$ ), consider the ellipsoid $\Omega=\mathscr{E}(P)$, with $P \in \mathbb{R}^{n \times n}$ and $P=P^{T}>0, H(j, J) \in \mathbb{R}^{1 \times n}$ such that $|H(j, J) x| \leq 1$ for every $J \subseteq \mathbb{N}_{m}$ and $j \in J, L(i, I(k)) \in \mathbb{R}^{1 \times n}$ such that $|L(i, I(k)) x| \leq 1$ for every $k \in \mathbb{N}_{m}$, every $I(k) \subseteq \mathbb{N}_{p}$ and $i \in I(k)$, for all $x \in \Omega$. Then we have $g(x) \in S(x)$ for all $x \in \Omega$, where

$$
S(x)=\operatorname{co}\left(\left\{Q(J, \mathbf{I}) x \in \mathbb{R}^{n}: J \subseteq \mathbb{N}_{m}, I(k) \subseteq \mathbb{N}_{p}, k \in \mathbb{N}_{m}\right\}\right),
$$

where $\mathbf{I}=\{I(1), I(2), \ldots, I(m)\}$ and

$$
\begin{aligned}
Q(J, \mathbf{I})= & A+\sum_{j \in \bar{J}} B_{(j)}\left(K_{j}+\sum_{i \in \bar{I}(j)} E_{j, i} F_{i}+\sum_{i \in I(j)} E_{j, i} L(i, I(j))\right) \\
& +\sum_{j \in J} B_{(j)} H(j, J) .
\end{aligned}
$$

Proof: Consider $x \in \Omega$. From Theorem 1 applied to the function given by $h(x)=K x+E \varphi(F x)$, we have that $h(x) \in$ $\operatorname{co}\left(\{M(I) x\}_{I}\right)$ for every $x \in \Omega$, where

$$
M(I)=K+\sum_{i \in \bar{I}} E_{(i)} F_{i}+\sum_{i \in I} E_{(i)} L(i, I),
$$

for every $I \subseteq \mathbb{N}_{p}$ and $\{M(I) x\}_{I}$ is introduced, for notational convenience, to denote the set $\left\{M(I) x: I \subseteq \mathbb{N}_{p}\right\}$. Then $g(x) \in$ $A x+B \varphi\left(\operatorname{co}\left(\{M(I) x\}_{I}\right)\right)$ and, for every $j \in \mathbb{N}_{m}$, we have that

$$
\begin{array}{r}
\varphi_{j}\left(\operatorname{co}\left(\{M(I) x\}_{I}\right)\right)=\left\{\varphi_{j}(T x): T x \in \operatorname{co}\left(\{M(I) x\}_{I}\right)\right\} \\
\subseteq\left\{\operatorname{co}\left(\left\{T_{j} x, H(j, J) x\right\}\right): T x \in \operatorname{co}\left(\{M(I) x\}_{I}\right)\right\},
\end{array}
$$


where the inclusion follows from the proof of Theorem 1 . Notice that $T x \in \operatorname{co}\left(\{M(I) x\}_{I}\right)$, for all $x \in \Omega$, is equivalent to say that there exists $\lambda_{I} \geq 0$, for every $I \subseteq \mathbb{N}_{p}$, such that $\sum_{I \subseteq \mathbb{N}_{p}} \lambda_{I}=1$ and $T x=\sum_{I \subseteq \mathbb{N}_{p}} \lambda_{I} M(I) x$ for all $x \in \mathbb{R}^{n}$, or, equivalently, $T_{j} x=\sum_{I \subseteq \mathbb{N}_{m}} \lambda_{I} M_{j}(I) x$ for all $j \in \mathbb{N}_{m}$. Then, for every $j \in \mathbb{N}_{m}$, $T x \in \operatorname{co}\left(\{M(I) x\}_{I}\right)$ implies $T_{j} x \in \operatorname{co}\left(\left\{M_{j}(I) x\right\}_{I}\right)$ and thus

$$
\begin{gathered}
\varphi_{j}\left(\operatorname{co}\left(\{M(I) x\}_{I}\right)\right) \subseteq \operatorname{co}\left(\left\{\operatorname{co}\left(\left\{M_{j}(I) x\right\}_{I}\right), H(j, J) x\right\}\right) \\
=\operatorname{co}\left(\left\{M_{j}\left(I_{0}\right) x, \ldots, M_{j}\left(I_{2^{p}}\right) x, H(j, J) x\right\}\right),
\end{gathered}
$$

where $I_{1}, I_{2}, \ldots, I_{2^{p}}$ denote, with a slight abuse of notation, the possible subsets of $\mathbb{N}_{p}$. Following the line of the proof of Theorem 1, it can be proven that, for every $j \in J$, every $x \in \Omega$ and every $\eta \in \mathbb{R}^{n}$, there exists a choice between $\eta^{T} B_{(j)} H(j, J) x$ and one element of $\left\{\eta^{T} B_{(j)} M_{j}(I) x: I \subseteq \mathbb{N}_{p}\right\}$ providing an upper bound of $\eta^{T} B_{(j)} \varphi_{j}\left(\operatorname{co}\left(\{M(I) x\}_{I}\right)\right)$. Then, for every $x \in \Omega$ and every $\eta \in \mathbb{R}^{n}$ there exists a selection of $J(x, \eta) \subseteq \mathbb{N}_{m}$ and $I(j, x, \eta) \subseteq \mathbb{N}_{p}$, with $j \in \mathbb{N}_{m}$, such that

$$
\begin{gathered}
\eta^{T} g(x) \leq \eta^{T} A x+\sum_{j \in \bar{J}(x, \eta)} \eta^{T} B_{(j)}\left(K_{j}+\sum_{i \in \bar{I}(j, x, \eta)} \eta^{T} E_{j, i} F_{i}\right. \\
\left.+\sum_{i \in I(j, x, \eta)} \eta^{T} E_{j, i} L(i, I(j, x, \eta))\right) x+\sum_{j \in J(x, \eta)} \eta^{T} B_{(j)} H(j, J(j, x, \eta)) x,
\end{gathered}
$$

and then, as for Theorem 1, we have that $g(x) \in S(x)$.

Notice that the bounding condition for nested saturations involves the existence of a set $I(k)$ for any $k \in \mathbb{N}_{m}$, besides of $J$. There are $2^{m}$ possible sets $J$ (each one representing a subset of $\mathbb{N}_{m}$ ) and $2^{p}$ possibilities of every $I(k)$, with $k \in \mathbb{N}_{m}$. Hence there are $2^{(p+1) m}$ different values of $Q(J, \mathbf{I})$, although some of them lead to redundant selections and could be discarded.

As shown in [13], applying Theorem 1 to continuous-time and discrete-time systems permits to recover or extend results presented in literature, for instance in [9], [10], [11].

\section{B. Quadratic stability for saturated hybrid systems}

The presented results are employed to state conditions for quadratic stability for hybrid systems with saturations, possibly nested. First the case of simple saturations (1)-(3) is considered. We impose the decreasing of the candidate Lyapunov function $V(x)=x^{T} P x$ along the continuous trajectories. Moreover, we have to ensure that the variation of $V(x)$ during a jump plus the variation during a flowing interval of $\rho$, is negative. This, with the temporal regularization, would imply that $V(x)$ is decreasing between two successive jumps. The resulting condition is less conservative than imposing the decreasing of $V(x)$ also during the jump. In the following, $m_{c}$ and $m_{d}$ are the number of columns of $\hat{B}$ and $\tilde{B}, p_{c}$ and $p_{d}$ those of $\hat{E}$ and $\tilde{E}$. Notice that the case of functions increasing along flow trajectories and decreasing during jumps, as well as more general cases, could be considered, see also [20].

Theorem 3: Given the hybrid system (1)-(3), consider the ellipsoid $\Omega=\mathscr{E}(P)$, with $P \in \mathbb{R}^{n \times n}$ and $P=P^{T}>0$, $\hat{H}(i, I) \in \mathbb{R}^{1 \times n}$ and $\tilde{H}(j, J) \in \mathbb{R}^{1 \times n}$ such that $|\hat{H}(i, I) x| \leq 1$ and $|\tilde{H}(j, J) x| \leq 1$, for all $x \in \Omega$, for every $I \subseteq \mathbb{N}_{m_{c}}$ and $i \in I$, $J \subseteq \mathbb{N}_{m_{d}}$ and $j \in J, \lambda>0$, and $\sigma \geq 0$. If

$$
\hat{N}(I)^{T} P+P \hat{N}(I) \leq-2 \lambda P,
$$

$$
\tilde{N}(J)^{T} e^{-\lambda \rho I_{n}} P e^{-\lambda \rho I_{n}} \tilde{N}(J)-\sigma M \leq P,
$$

where $\hat{N}(I)$ and $\tilde{N}(J)$ are defined as

$$
\begin{aligned}
& \hat{N}(I)=\hat{A}+\sum_{i \in \bar{I}} \hat{B}_{(i)} \hat{K}_{i}+\sum_{i \in I} \hat{B}_{(i)} \hat{H}(i, I), \\
& \tilde{N}(J)=\tilde{A}+\sum_{j \in \bar{J}} \tilde{B}_{(j)} \tilde{K}_{j}+\sum_{j \in J} \tilde{B}_{(j)} \tilde{H}(j, J),
\end{aligned}
$$

for all $I \subseteq \mathbb{N}_{m_{c}}$ and $J \subseteq \mathbb{N}_{m_{d}}$, then $\Omega$ is an ellipsoidal estimation of the domain of attraction and a local Lyapunov function in $\Omega$ for the hybrid system (1)-(3) can be determined.

Proof: Notice that, from temporal regularization, the system can flow for all $x \in \Omega$. Condition (8) implies that $V(x)$ decreases along the continuous-time trajectories, within the whole set $\Omega$. In fact, from Theorem 1 we have that $\hat{g}(x) \in \hat{G}(x)$, with $\hat{G}(x)=\operatorname{co}\left(\left\{\hat{N}(I) x \in \mathbb{R}^{n}: I \subseteq \mathbb{N}_{m_{c}}\right\}\right)$, which implies the existence of $I(x, \eta) \subseteq \mathbb{N}_{m_{c}}$ such that $\eta^{T} \hat{g}(x) \leq \eta^{T} \hat{N}(I(x, \eta)) x$, for all $\eta \in \mathbb{R}^{n}$, as shown in the proof of Theorem 1, see (7). Posing $\eta^{T}=x^{T} P$ and from (8), we have that

$$
\begin{aligned}
\dot{V}(x) & =\frac{\partial V(x)}{\partial x} \dot{x}=x^{T} P \dot{x}+\dot{x}^{T} P x=x^{T} P \hat{g}(x)+\hat{g}(x)^{T} P x \\
& \leq-x^{T} \lambda P x-x^{T} P \lambda x,
\end{aligned}
$$

with $\dot{x}=\hat{g}(x)$ as in (1). Consider the system $\dot{\bar{x}}=-\lambda \bar{x}$, whose trajectories are given by $\bar{x}(t)=e^{-\lambda t I_{n}} \bar{x}(0)$. The time-derivative of $V(x)$ along the trajectories of system $\dot{\bar{x}}=-\lambda \bar{x}$ is given by

$$
\dot{\bar{V}}(\bar{x})=\frac{\partial V(\bar{x})}{\partial \bar{x}} \dot{\bar{x}}=\bar{x}^{T} P \dot{\bar{x}}+\dot{\bar{x}}^{T} P \bar{x}=-\bar{x}^{T} \lambda P \bar{x}-\bar{x}^{T} P \lambda \bar{x},
$$

for every $\bar{x} \in \mathbb{R}^{n}$. Then, for every initial condition $x(0)=\bar{x}(0) \in$ $\Omega$, the time-derivatives along the trajectories of systems (1) and $\dot{\bar{x}}=-\lambda \bar{x}$ are such that $\dot{V}(x)-\dot{\bar{V}}(\bar{x}) \leq 0$. Since the integral of a non-positive function is smaller or equal than 0 , we have

$$
\begin{aligned}
& V(x(\tau))-V(x(0))=\int_{0}^{\tau} \dot{V}(x(t)) d t \leq \int_{0}^{\tau} \dot{\bar{V}}(\bar{x}) d t \\
& =\int_{0}^{\tau} \dot{\bar{x}}^{T} P \bar{x} d t+\int_{0}^{\tau} \bar{x}^{T} P \dot{\bar{x}} d t=\bar{x}(\tau)^{T} P \bar{x}(\tau)-\bar{x}(0)^{T} P \bar{x}(0),
\end{aligned}
$$

and then, for $x(0)=\bar{x}(0)$, we have that

$$
V(x(\tau)) \leq \bar{x}(\tau)^{T} P \bar{x}(\tau)=x(0)^{T} e^{-\lambda \tau I_{n}} P e^{-\lambda \tau I_{n}} x(0) .
$$

Thus the trajectories of systems (1) and $\dot{\bar{x}}=-\lambda \bar{x}$ starting at the same point $x(0)$ and after time $\tau$ are such that $V(x(\tau)) \leq$ $V(\bar{x}(\tau))$, for every $\tau \geq 0$. Now we can prove that conditions (8) and (9) imply that the value of $V(x)$ decreases between two jumping instant. For the temporal regularization, it is sufficient to prove that the variation (possibly positive) of function $V(x)$ during a jump plus the variation of $V(x)$ after $\rho$ of flowing is non-positive. Such condition must be verified when the state is in the jump set $\mathscr{J}$. Consider a jump at time 0 (no loss of generality is introduced) followed by a flowing interval of duration $\rho$. Denote with $x_{0}^{-}$the state before the jump and $x_{0}^{+}$ the state after the jump. Then the condition reads

$$
V\left(x_{0}^{+}\right)-V\left(x_{0}^{-}\right)+\int_{0^{+}}^{\rho} \dot{V}(x) d t \leq 0,
$$


for all $x_{0}^{-} \in \mathscr{J}$, and thus, being $V\left(x_{0}^{+}\right)=\left(x_{0}^{+}\right)^{T} P x_{0}^{+}$, we have

$$
x(\rho)^{T} P x(\rho) \leq\left(x_{0}^{-}\right)^{T} P x_{0}^{-} .
$$

This has a clear geometrical meaning, as (12) is equivalent to require that $V(x)$ before the jump is greater than (or equal to) its value after time $\rho$, when a successive jump could occur. From (11), with $x(0)=x_{0}^{+}$, we have that if $\left(x_{0}^{+}\right)^{T} e^{-\lambda \rho I_{n}} P e^{-\lambda \rho I_{n}} x_{0}^{+} \leq\left(x_{0}^{-}\right)^{T} P x_{0}^{-}$, or equivalently

$$
\tilde{g}\left(x_{0}^{-}\right)^{T} P \tilde{g}\left(x_{0}^{-}\right)=\left(x_{0}^{+}\right)^{T} P x_{0}^{+} \leq\left(x_{0}^{-}\right)^{T} e^{\lambda \rho I_{n}} P e^{\lambda \rho I_{n}} x_{0}^{-},
$$

holds, then condition (12) is satisfied.

Considering the discrete-time dynamics, we have that $\tilde{g}(x) \in$ $\tilde{G}(x)$, with $\tilde{G}(x)=\operatorname{co}\left(\left\{\tilde{N}(J) x \in \mathbb{R}^{n}: J \subseteq \mathbb{N}_{m_{d}}\right\}\right)$, from Theorem 1. The quadratic function $V(x)=x^{T} P x$, is convex and bounded over $\mathbb{R}^{n}$. Since the supremum of a convex function relative to a convex, compact set $C$ is attained at some extreme of $C$, see [18], then there is an extreme of set $\tilde{G}(x)$ where the maximum of $V(x)$ is attained. Therefore there exists $J(x) \subseteq \mathbb{N}_{m_{d}}$ such that

$$
\tilde{g}(x)^{T} P \tilde{g}(x) \leq x^{T} \tilde{N}(J(x))^{T} P \tilde{N}(J(x)) x=\sup _{y \in \tilde{G}(x)} V(y) .
$$

Then condition (9) implies (13) for all $x \in \Omega \cap \mathscr{J}$ and $\tau \leq \rho$. In fact, posing $x=x_{0}^{-}$and applying the S-procedure, we have

$$
\begin{aligned}
\left(x^{+}\right)^{T} P x^{+} & =\tilde{g}(x)^{T} P \tilde{g}(x) \leq x^{T} \tilde{N}(J(x))^{T} P \tilde{N}(J(x)) x \\
& \leq x^{T} e^{\lambda \rho I_{n}}(P+\sigma M) e^{\lambda \rho I_{n}} x,
\end{aligned}
$$

that leads to satisfaction of condition (13), and hence (12), for every $x \in \Omega$ such that $x \in \mathscr{J}$.

A condition for global asymptotic stability is stated for hybrid systems (1)-(3).

Corollary 1: Given the hybrid system (1)-(3) and $P \in \mathbb{R}^{n \times n}$ with $P=P^{T}>0, \lambda>0$ and $\sigma \geq 0$. If (8) and (9) hold with

$$
\hat{N}(I)=\hat{A}+\sum_{i \in \bar{I}} \hat{B}_{(i)} \hat{K}_{i}, \quad \tilde{N}(J)=\tilde{A}+\sum_{j \in \bar{J}} \tilde{B}_{(j)} \tilde{K}_{j},
$$

for every $I \subseteq \mathbb{N}_{m_{c}}$ and $J \subseteq \mathbb{N}_{m_{d}}$, then a global Lyapunov function for the hybrid system (1)-(3) can be determined.

Proof: The result follows from Theorem 3 with $\hat{H}(i, I)=$ $\tilde{H}(j, J)=0_{1 \times n}$, for all $I \subseteq \mathbb{N}_{m_{c}}, J \subseteq \mathbb{N}_{m_{d}}, i \in I$ and $j \in J$.

Notice that asymptotic stability of the systems $\dot{x}=\hat{A} x$ and $x^{+}=\tilde{A} x$ is a necessary condition for global asymptotic stability of system (1)-(3), in fact, given by (8) and (9) with $I=\mathbb{N}_{m_{c}}$ (then $\bar{I}=\emptyset$ ) and $J=\mathbb{N}_{m_{d}}$ (thus $\bar{J}=\emptyset$ ) in (15). Also asymptotic stability of $\dot{x}=(\hat{A}+\hat{B} \hat{K}) x$ and $x^{+}=(\tilde{A}+\tilde{B} \tilde{K}) x$, implied by conditions (8) and (9) with $I=\emptyset$ and $J=\emptyset$ in (15), is necessary. Analogous results for the case of nested saturations (3)-(5) are stated in the following theorem.

Theorem 4: Given the hybrid system with nested saturations (3)-(5), consider the ellipsoid $\Omega=\mathscr{E}(P)$, with $P \in \mathbb{R}^{n \times n}$ and $P=P^{T}>0, \lambda>0$ and $\sigma \geq 0$. Assume there exist: $\hat{H}(j, J) \in \mathbb{R}^{1 \times n}$ such that $|\hat{H}(j, J) x| \leq 1$ for every $J \subseteq \mathbb{N}_{m_{c}}$ and $j \in J ; \hat{L}(i, I(k)) \in \mathbb{R}^{1 \times n}$ such that $|\hat{L}(i, I(k)) x| \leq 1$ for every $k \in \mathbb{N}_{m_{c}}$, every $I(k) \subseteq \mathbb{N}_{p_{c}}$ and $i \in I(k)$, for all $x \in \Omega$; $\tilde{H}(u, U) \in \mathbb{R}^{1 \times n}$ such that $|\tilde{H}(u, U) x| \leq 1$ for every $U \subseteq \mathbb{N}_{m_{d}}$ and $u \in U ; \tilde{L}(v, V(l)) \in \mathbb{R}^{1 \times n}$ such that $|\tilde{L}(v, V(l)) x| \leq 1$ for every $l \in \mathbb{N}_{m_{d}}$, every $V(l) \subseteq \mathbb{N}_{p_{d}}$ and $v \in V(l)$, for all $x \in \Omega$, such that:

$$
\hat{Q}(J, \mathbf{I})^{T} P+P \hat{Q}(J, \mathbf{I}) \leq-2 \lambda P,
$$

$$
\tilde{Q}(U, \mathbf{V})^{T} e^{-\lambda \rho I_{n}} P e^{-\lambda \rho I_{n}} \tilde{Q}(U, \mathbf{V})-\sigma M \leq P,
$$

with $\mathbf{I}=\left\{I(1), \ldots, I\left(m_{c}\right)\right\}$ and $\mathbf{V}=\left\{V(1), \ldots, V\left(m_{d}\right)\right\}$, where $\hat{Q}(J, \mathbf{I})$ and $\tilde{Q}(U, \mathbf{V})$ are defined as

$$
\begin{aligned}
\hat{Q}(J, \mathbf{I})= & \hat{A}+\sum_{j \in \bar{J}} \hat{B}_{(j)}\left(\hat{K}_{j}+\sum_{i \in \bar{I}(j)} \hat{E}_{j, i} \hat{F}_{i}\right. \\
& \left.+\sum_{i \in I(j)} \hat{E}_{j, i} \hat{L}(i, I(j))\right)+\sum_{j \in J} \hat{B}_{(j)} \hat{H}(j, J), \\
\tilde{Q}(U, \mathbf{V})= & \tilde{A}+\sum_{u \in \bar{U}} \tilde{B}_{(u)}\left(\tilde{K}_{u}+\sum_{v \in \bar{V}(u)} \tilde{E}_{u, v} \tilde{F}_{v}\right. \\
& \left.+\sum_{v \in V(u)} \tilde{E}_{u, v} \tilde{L}(v, V(u))\right)+\sum_{u \in U} \tilde{B}_{(u)} \tilde{H}(u, U),
\end{aligned}
$$

for all $J \subseteq \mathbb{N}_{m_{c}}, I(k) \subseteq \mathbb{N}_{p_{c}}, k \in \mathbb{N}_{m_{c}}$ and all $U \subseteq \mathbb{N}_{m_{d}}$, $V(l) \subseteq \mathbb{N}_{p_{d}}, l \in \mathbb{N}_{m_{d}}$. Then $\Omega$ is an ellipsoidal estimation of the domain of attraction and a local Lyapunov function in $\Omega$ for the hybrid system (3)-(5) can be determined.

Furthermore, a global Lyapunov function for the hybrid system with nested saturations (3)-(5) can be determined if conditions (16)-(17) hold with

$$
\begin{aligned}
& \hat{Q}(J, \mathbf{I})=\hat{A}+\sum_{j \in \bar{J}} \hat{B}_{(j)}\left(\hat{K}_{j}+\sum_{i \in \bar{I}(j)} \hat{E}_{j, i} \hat{F}_{i}\right), \\
& \tilde{Q}(U, \mathbf{V})=\tilde{A}+\sum_{u \in \bar{U}} \tilde{B}_{(u)}\left(\tilde{K}_{u}+\sum_{v \in \bar{V}(u)} \tilde{E}_{u, v} \tilde{F}_{v}\right),
\end{aligned}
$$

for all $J \subseteq \mathbb{N}_{m_{c}}, I(k) \subseteq \mathbb{N}_{p_{c}}, k \in \mathbb{N}_{m_{c}}$ and all $U \subseteq \mathbb{N}_{m_{d}}$, $V(l) \subseteq \mathbb{N}_{p_{d}}, l \in \mathbb{N}_{m_{d}}$, where $\mathbf{I}=\left\{I(1), I(2), \ldots, I\left(m_{c}\right)\right\}$ and $\mathbf{V}=\left\{V(1), V(2), \ldots, V\left(m_{d}\right)\right\}$.

Proof: This result can be proved by using reasonings analogous to those of Theorem 3 and Corollary 1 and employing the results from Theorem 2 .

Remark 2: Function $V(x)$ in Theorems 3 and 4 and Corollary 1 are not necessarily decreasing along the trajectories of systems (1)-(3) and (3)-(5), due to jumps. However, $V(x)$ can be used to determine Lyapunov functions for the saturated hybrid systems.

\section{Computational issues}

Some computation oriented considerations on how to practically obtain a quadratic Lyapunov function for systems (1)-(3) and (3)-(5) are provided. First, we propose a formulation of the condition provided by Theorem 3 which can be reduced in LMI form by fixing the value of $\lambda$.

Proposition 1: Consider the hybrid system (1)-(3). Suppose that there exist $W \in \mathbb{R}^{n \times n}$ with $W=W^{T}>0, \lambda>0, \hat{Z}(i, I) \in$ $\mathbb{R}^{1 \times n}$ and $\tilde{Z}(j, J) \in \mathbb{R}^{1 \times n}$ for every $I \subseteq \mathbb{N}_{m_{c}}, i \in I, J \subseteq \mathbb{N}_{m_{d}}$ and $j \in J$, such that conditions

$$
\begin{gathered}
\left(\hat{A} W+\sum_{i \in \bar{I}} \hat{B}_{(i)} \hat{K}_{i} W+\sum_{i \in I} \hat{B}_{(i)} \hat{Z}(i, I)+\lambda W\right)+\left(W \hat{A}^{T}\right. \\
\left.+\sum_{i \in \bar{I}} W \hat{K}_{i}^{T} \hat{B}_{(i)}^{T}+\sum_{i \in I} \hat{Z}(i, I)^{T} \hat{B}_{(i)}^{T}+\lambda W\right) \leq 0, \\
{\left[\begin{array}{cc}
W & \left(W \tilde{A}^{T}+\sum_{j \in \bar{J}} W \tilde{K}_{j}^{T} \tilde{B}_{(j)}^{T}+\sum_{j \in J} \tilde{Z}(j, J)^{T} \tilde{B}_{(j)}^{T}\right) e^{-\lambda \rho I_{n}} \\
* & W
\end{array}\right] \geq 0,} \\
{\left[\begin{array}{cc}
1 & \hat{Z}(i, I) \\
* & W
\end{array}\right] \geq 0, \forall i \in I, \quad\left[\begin{array}{cc}
1 & \tilde{Z}(j, J) \\
* & W
\end{array}\right] \geq 0, \forall j \in J,}
\end{gathered}
$$


are satisfied for every $I \subseteq \mathbb{N}_{m_{c}}$ and $J \subseteq \mathbb{N}_{m_{d}}$. Then set $\Omega=$ $\mathscr{E}(P)$, with $P=W^{-1}$, is an ellipsoidal estimation of the domain of attraction and a local Lyapunov function in $\Omega$ for the hybrid system (1)-(3) can be determined.

Proof: The proposition stems from Theorem 3. In fact, it can be proved, using standard matrix inequalities manipulation techniques, that (20)-(22) imply the conditions of the theorem, with $W=P^{-1}, \hat{Z}(i, I)=\hat{H}(i, I) W$ and $\tilde{Z}(j, J)=\tilde{H}(j, J) W$, for every $I \subseteq \mathbb{N}_{m_{c}}$ and $i \in I, J \subseteq \mathbb{N}_{m_{d}}$ and $j \in J$. The only difference is that condition (9), concerning $x \in \Omega$ and $(x, \tau) \in \mathscr{J}$, is relaxed in (21) imposing the condition on jumps for all $x \in \Omega$. Finally, (22) assures that $|\hat{H}(i, I) x| \leq 1$ and $|\tilde{H}(j, J) x| \leq 1$, for all $x \in \Omega$, every $I \subseteq \mathbb{N}_{m_{c}}$ and $J \subseteq \mathbb{N}_{m_{d}}$.

Notice that although $V(x)$ in Proposition 1 do not decrease along the trajectories, Lyapunov functions can be determined.

Remark 3: As stated in the proof of Proposition 1, the condition on the variation of the value of $V(x)$ during the jump is imposed over the whole set $\Omega$, although it could have been restricted to the set $\mathscr{J}$. In fact, the term $\sigma M$ in (9) is not present in (21). This yields some conservativeness, but permits to pose the problem in LMI form, fixing $\lambda$. Removing this source of conservativeness is a possible future improvement.

The result provided in Proposition 1 can be used to pose an optimization problem to maximize the size of $\Omega$ and hence to provide a solution to Problem 1.

Remark 4: A possible evaluation criterion is the maximization of the value of $\beta$ such that the polytope $\beta L=\operatorname{co}(\{\beta v(k) \in$ $\left.\left.\mathbb{R}^{n}: k \in \mathbb{N}_{V}\right\}\right)$ is contained in the estimate $\Omega=\mathscr{E}(P)$, where $v(k) \in \mathbb{R}^{n}$, with $k \in \mathbb{N}_{V}$, are given points in the state space. The optimization problem results:

$$
\begin{aligned}
& \max _{\beta, \lambda, \tilde{Z}, \hat{z}, W} \beta \\
& \text { s.t. } \quad(20),(21),(22), \quad \forall I \subseteq \mathbb{N}_{m_{c}}, \quad \forall J \subseteq \mathbb{N}_{m_{d}} \\
& {\left[\begin{array}{cc}
1 & \beta v(k)^{T} \\
* & W
\end{array}\right] \geq 0, \quad \forall k \in \mathbb{N}_{V},}
\end{aligned}
$$

where, for sake of notational compactness, we denoted with $\tilde{Z}$ and $\hat{Z}$ the matrices $\hat{Z}(i, I)$ and $\tilde{Z}(j, J)$ for all $I \subseteq \mathbb{N}_{m_{c}}$ and $i \in I, J \subseteq \mathbb{N}_{m_{d}}$ and $j \in J$. Constraints (20)-(22) ensure that $V(x)=x^{T} P x$ yields a Lyapunov function in $\mathscr{E}\left(W^{-1}\right)$ for the hybrid system, and the second set of constraints imposes that $\beta v(k) \in \mathscr{E}\left(W^{-1}\right)$, for every $k \in \mathbb{N}_{V}$.

Notice that, although the constraints (20) and (21) are not linear in the optimization variables, they are LMI for a fixed $\lambda$. Then, the problem can be solved for different values of $\lambda>0$, to obtain a guess of the maximal value of $\beta$. Notice also that $\lambda$ is a bound on the decreasing rate of the quadratic function along the trajectories of the continuous-time dynamics, then it could be considered as a design parameter and fixed beforehand. The LMI condition for global asymptotic stability for system (1)-(3) (and fixed $\lambda$ ) follows.

Corollary 2: Consider the hybrid system (1)-(3), matrix $P \in$ $\mathbb{R}^{n \times n}$ with $P=P^{T}>0, \lambda>0$ and $\sigma \geq 0$. If conditions

$$
\begin{gathered}
\left(\hat{A}+\sum_{i \in \bar{I}} \hat{B}_{(i)} \hat{K}_{i}\right)^{T} P+P\left(\hat{A}+\sum_{i \in \bar{I}} \hat{B}_{(i)} \hat{K}_{i}\right) \leq-2 \lambda P, \\
\left(\tilde{A}+\sum_{j \in \bar{J}} \tilde{B}_{(j)} \tilde{K}_{j}\right)^{T} e^{-\lambda \rho I_{n}} P e^{-\lambda \rho I_{n}}\left(\tilde{A}+\sum_{j \in \bar{J}} \tilde{B}_{(j)} \tilde{K}_{j}\right)-\sigma M \leq P,
\end{gathered}
$$

hold for all $I \subseteq \mathbb{N}_{m_{c}}$ and $J \subseteq \mathbb{N}_{m_{d}}$, then $V(x)=x^{T} P x$ yields a global Lyapunov function for the hybrid system (1)-(3).

Remark 5: The conditions for hybrid systems with nested saturations (3)-(5) can be easily recovered, by properly modifying terms $\hat{B}_{(i)} \hat{K}_{i} W$ in (20) and $\tilde{B}_{(i)} \tilde{K}_{i} W$ in (21), as well terms $\hat{B}_{(i)} \hat{K}_{i}$ and $\tilde{B}_{(j)} \tilde{K}_{j}$ in (24).

\section{NUMERICAL EXAMPLES}

The systems presented below can be expressed as in (1)-(2), or (4)-(5), by posing $x=\left(x_{p}, x_{c}\right)$.

Example 1: Consider the linear unstable system, proposed in [15], in closed-loop with a stabilizing reset PI controller:

$$
\left\{\begin{array}{l}
\dot{x}_{p}=0.1 x_{p}+\varphi\left(y_{c}\right), \quad\left\{\begin{array}{l}
\dot{x}_{c}=-0.2 y_{p}, \\
y_{p}=x_{p},
\end{array}=x_{c}-2 y_{p} .\right.
\end{array}\right.
$$

The dynamics characterizing the reset behavior with saturation is $x_{c}^{+}=x_{c}+\varphi\left(-x_{c}\right)$. The minimum time interval between two jumps is set to 2 seconds, that is $\rho=2$.

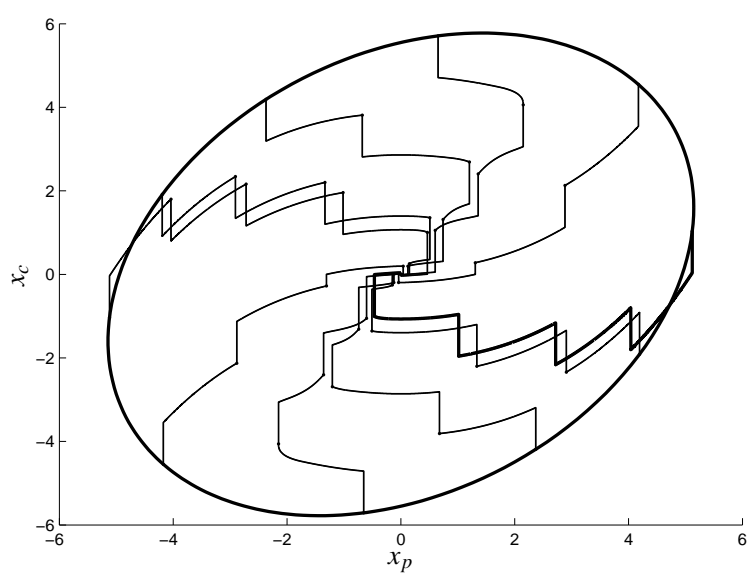

Fig. 1. Set $\Omega$ and trajectories of the saturated reset system.

We solve the optimization problem (23) where points $v(k)$, with $k \in \mathbb{N}_{4}$, are the vertices of the square set $L=\left\{x \in \mathbb{R}^{2}\right.$ : $\left.\|x\|_{\infty} \leq 1\right\}$, and for different values of $\lambda$. We found that the value of $\lambda=0.02$ provides the best value (among those tested) of $\beta$, that is $\beta=3.2689$ with

$$
P=\left[\begin{array}{cc}
0.0409 & -0.0101 \\
* & 0.03241
\end{array}\right] .
$$

The set $\Omega=\mathscr{E}(P)$ is an estimation of the domain of attraction, regardless of the set $\left\{x \in \mathbb{R}^{n}: x^{T} M x \geq 0\right\}$. In Figure $1, \Omega$ is depicted with some trajectories of the system assuming that the jump can occur at any $x \in \Omega$. Notice in particular the trajectory marked in bold line with initial condition $x(0)=x_{0}^{-}=$ $\left[\begin{array}{lll}5.1188 & 1.0376\end{array}\right]^{T}$. With the first jump at time 0 the trajectory leaves $\Omega$, then $V(x)$ increases, i.e. $V\left(x_{0}^{+}\right)=1.0686>1$. At the time of the second jump the state is contained in the ellipsoid, with $V\left(x\left(\rho^{-}\right)\right)=0.9196<1$. Then $V(x)$ decreases between the two jumps, as ensured by Theorem 3 .

Example 2: The case of nested saturations is considered. A further saturation is added between the plant output and the controller input of the continuous-time system (25):

$$
\left\{\begin{aligned}
\dot{x}_{p} & =0.1 x_{p}+\varphi\left(x_{c}-2 \varphi\left(x_{p}\right)\right) \\
\dot{x}_{c} & =-0.2 \varphi\left(x_{p}\right)
\end{aligned}\right.
$$


while the discrete-time behavior is as in Example 1. The solution of the optimization problem (23) adapted to nested saturations and with $\lambda=0.02$ leads to $\beta=1.8922$. As expected, the further saturation entails a reduction of the size of the estimation of the domain of attraction, see Figure 2.

Example 3: The condition for global asymptotic stability provided by Corollary 2 is applied to a multi-input system. Consider the system, inspired to the examples in work [21] and references therein, whose dynamics are given by

$$
A_{p}=\left[\begin{array}{cc}
-4 & 1 \\
0 & -1
\end{array}\right], \quad B_{p}=\left[\begin{array}{ll}
1 & 3 \\
3 & 1
\end{array}\right], \quad C_{p}=\left[\begin{array}{ll}
4 & 0
\end{array}\right],
$$

in closed-loop with continuous-time dynamical controller whose matrices are

$$
A_{c}=-3, B_{c}=-1, C_{c}=\left[\begin{array}{c}
0.1 \\
0.22
\end{array}\right], D_{c}=\left[\begin{array}{c}
-0.0625 \\
-0.1250
\end{array}\right] \text {. }
$$

The controller discrete-time dynamics is a saturated reset, i.e. $x_{c}^{+}=x_{c}+\varphi\left(-x_{c}\right)$, and the plant state performs an instantaneous rotation of $\pi / 4$ radians at any jump instant. Notice that asymptotic stability of both the open-loop and closed-loop continuous-time systems in absence of saturation, necessary conditions for global asymptotic stability, are ensured. Posing $\rho=0.5$ and $\lambda=0.01$ and assuming that the jump can occur at any $x \in \mathbb{R}^{n}$, conditions (24) are satisfied by

$$
P=\left[\begin{array}{ccc}
2.0972 & 0.0068 & -0.0113 \\
* & 2.1054 & -0.0056 \\
* & * & 1.8822
\end{array}\right],
$$

for every $I \subseteq \mathbb{N}_{m_{c}}$ and $J \subseteq \mathbb{N}_{m_{d}}$. Then, from Corollary 2, the saturated reset system is globally asymptotically stable and $V(x)=x^{T} P x$ yields a global Lyapunov function.

\section{Conclusions}

In this paper we dealt with the problems of characterizing quadratic stability and computing ellipsoidal estimations of the domain of attraction for saturated hybrid systems. The results presented are based on a geometrical approach to the analysis of saturated functions, also in case of nested saturations, which

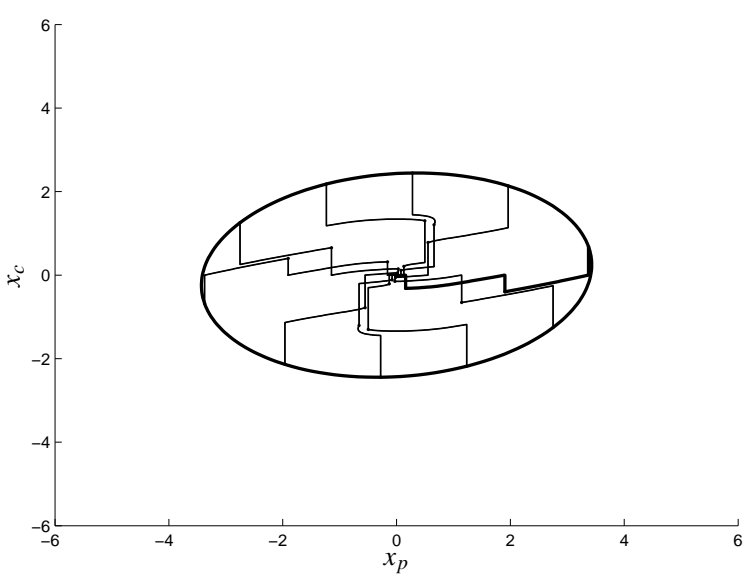

Fig. 2. Set $\Omega$ and trajectories of the reset system with nested saturations. permitted to formulate contractiveness conditions of ellipsoids for a rather generic class of saturated hybrid systems.

An interesting forthcoming issue could be to exploit the hybrid loop to improve the performance of a controlled system in presence of exogenous signals. This could be achieved by designing the reset law and both the flow and jump sets.

\section{REFERENCES}

[1] M. S. Branicky, V. S. Borkar, and S. K. Mitter, "A unified framework for hybrid control: model and optimal control theory," IEEE Transactions on Automatic Control, vol. 43, pp. 31-45, 1998.

[2] D. Liberzon, Switching in systems and control. Boston: Birkhauser, 2003.

[3] R. Goebel, J. P. Hespanha, A. R. Teel, C. Cai, and R. Sanfelice, "Hybrid Systems: Generalized solutions and robust stability," Proc. IFAC: Symp on Nonlinear Control Systems, Stuttgart, Germany, pp. 1-12, 2004.

[4] Z. Sun and S. S. Ge, Switched linear systems: control and design. London: Springer-Verlag, 2005.

[5] R. Goebel, R. Sanfelice, and A. R. Teel, "Hybrid dynamical systems," IEEE Control Systems Magazine, vol. 29, no. 2, pp. 28-93, 2009.

[6] M. Lazar and W. P. M. H. Heemels, "Predictive control of hybrid systems: Input-to-state stability results for sub-optimal solutions," Automatica, vol. 45, no. 1, pp. 180-185, 2009.

[7] C. Prieur, R. Goebel, and A. R. Teel, "Hybrid feedback control and robust stabilization of nonlinear systems," IEEE Transactions on Automatic Control, vol. 52, no. 11, pp. 2103-2117, 2007.

[8] J. M. Gomes da Silva Jr. and S. Tarbouriech, "Local stabilization of discrete-time linear systems with saturating controls: An LMI-based approach," IEEE Transactions on automatic control, vol. 46, pp. 119$125,2001$.

[9] T. Hu and Z. Lin, "Exact characterization of invariant ellipsoids for single input linear systems subject to actuator saturation," IEEE Transactions on Automatic Control, vol. 47, no. 1, pp. 164-169, jan. 2002.

[10] T. Hu, Z. Lin, and B. M. Chen, "Analysis and design for discrete-time linear systems subject to actuator saturation," Systems \& Control Letters, vol. 45, no. 2, pp. 97-112, 2002.

[11] T. Alamo, A. Cepeda, and D. Limon, "Improved computation of ellipsoidal invariant sets for saturated control systems," in Proc. 44th IEEE Conf. on Decision and Control and European Control Conference CDC-ECC 2005, Seville, Spain, Dec. 2005, pp. 6216-6221.

[12] T. Alamo, A. Cepeda, D. Limon, and E. F. Camacho, "A new concept of invariance for saturated systems," Automatica, vol. 42, pp. 1515-1521, 2006.

[13] M. Fiacchini, S. Tarbouriech, and C. Prieur, "Ellipsoidal invariant sets for saturated hybrid systems," in American Control Conference, 2011. ACC '11, San Francisco, CA, USA, June 2011.

[14] R. L. Grossmann, A. Nerode, A. P. Ravn, and H. Rischel, "Hybrid systems." Lecture Notes in Computer Science, vol. 736, 1993.

[15] S. Tarbouriech, T. Loquen, and C. Prieur, "Anti-windup strategy for reset control systems," International Journal of Robust and Nonlinear Control, vol. 21, no. 10, pp. 1159-1177, 2011.

[16] D. Nesic̀, L. Zaccarian, and A. R. Teel, "Stability properties of reset systems," Automatica, vol. 44, no. 8, pp. 2019-2026, 2008.

[17] S. Tarbouriech, C. Prieur, and J. M. G. da Silva Jr., "Stability analysis and stabilization of systems presenting nested saturations," IEEE Transactions on Automatic Control, vol. 51, no. 8, pp. 1364-1371, 2006.

[18] R. T. Rockafellar, Convex analysis. Princeton University Press, USA, 1970.

[19] R. Schneider, Convex bodies: The Brunn-Minkowski theory. England: Cambridge University Press, 1993, vol. 44.

[20] J. P. Hespanha, D. Liberzon, and A. R. Teel, "Lyapunov conditions for input-to-state stability of impulsive systems," Automatica, vol. 44, no. 11 , pp. $2735-2744$.

[21] O. Beker, C. V. Hollot, Y. Chait, and H. Han, "Fundamental properties of reset control systems," Automatica, vol. 40, pp. 905-915, 2004. 